1. Bei Anwendung von Methylorange und Phenolphtalein:

a) Die Phosphorsäure muß entfernt werden.

b) Die Berechnung der Borsäure erfolgt mit Hilfe des theoretischen Faktors: $1 \mathrm{ccm}$ Normallauge entspricht $62 \mathrm{mg}$ Borsäure.

2. Bei Anwendung von Phenolphtalein allein:

a) Die Phosphorsäure braucht nicht entfernt zu werden.

b) Für die Berechnung ist diejenige Alkalimenge zugrunde zu legen, welche von der in wässeriger Lösung bereits neutralisierten Flüssigkeit nach dem Glycerinzusatz verbraucht wird ${ }^{1}$ ). Der von jedem Analytiker genauer zu ermittelnde Wert ist von der Menge der vorhandenen Borsäure und der Konzentration der Lösung abhängig und beträgt unter den angegebenen Verhältnissen für $1 \mathrm{ccm}$ Normallauge bei Anwesenheit von:

$0,1000 \mathrm{~g}$ Borsäure in $50 \mathrm{ccm}$ etwa $63,29 \mathrm{mg} \mathrm{H}_{3} \mathrm{BO}_{3}$ (B eythien u. Hempel)

$0,1558, n \pi n, \quad$ n 64,39 n,$\quad$ (Lührig)

0,4000 , $, ", \quad 66,67, \quad$ (Beythien u. Hempel)

c) Der von Jörgensen angegebene Titer von $73 \mathrm{mg}$, als Mittelwert von 20 sehr stark abweichenden Bestimmungen, ist zu verwerfen.

Dresden, im Juni 1905.

1) Dieser Punkt ist von K, Wind isch außer acht gelassen worden.

\title{
Über die Bestimmung des Fettgehaltes der Butter nach Gottlieb.
}

\author{
Von \\ Dr. Anton Burr.
}

Mitteilung aus der Versuchsstation für Molkereiwesen in Kiel.

Für die Bestimmung des Fettes in der Milch stehen uns genügend handliche und sichere Verfahren zur Verfügung, bei denen man entweder 1. das Volumen der Fettschicht direkt abliest oder 2. aus dem spezifischen Gewicht oder dem Brechungsindex einer Ätherfett-Lösung den Gehalt an Fett ersieht oder 3..das Fett durch ein Extraktionsmittel der Milch entzieht, dieses verdunstet, und das Fett trocknet und wägt. Unter den letzten Verfahren ist ganz besonders die Gottlieb'sche Fettbestimmung hervorzuheben, die mit bequemer Handlichkeit eine sehr große Genauigkeit verbindet und sowohl für Untersuchung von Rahm, Voll-, Mager- und Buttermilch und Molken auf Fett, als auch zur Bestimmung desselben in Käse ${ }^{1}$ ) verwendet wird.

Ganz anders liegen die Verhältnisse bei Butter, für die bisher nur recht wenige Verfahren im Gebrauch sind, die teilweise ungenaue und nur annähernde Ergebnisse liefern, wie z. B. die Fettbestimmung in Butter nach Gerber, teilweise wohl ganz gute Ergebnisse bringen, aber sehr umständlich sind und zur Ausführung viel Zeit erfordern. Die bekanntesten beruhen auf der Extraktion des Fettes aus der durch

1) Vergl. E. Ratzlaff, Milch-Ztg. 1903, 32, 65; diese Zeitschrift 1904, 7, 409. 
Trocknen wasserfrei gemachten Butter mit einem Extraktionsmittel, Verdunsten desselben und Wägen des Fettes. Entweder zieht man die bei der Wasserbestimmung in Butter zurückbleibende Masse im Soxhlet'schen Extraktionsapparate durch wasserfreien Äther oder Petroläther aus, oder man entfettet sie auf einem Filter mit genannten Reagentien bis zur Erschöpfung. In beiden Fällen wird das Fett nach Verdunsten des Lösungsmittels und Trocknen bis zur Gewichtskonstanz gewogen. Die "Vereinbarungen" ${ }^{\text {) }}$ ) geben folgende Anweisung zur Bestimmung des Fettes in der Butter:

1. Indirekt; durch Subtraktion des Gehaltes an (Wasser + Kasein + Milchzucker + Salzen) von 100 .

2. Direkt, und zwar:

a) Durch Eindampfen eines aliquoten Teiles der bei der Bestimmung von (Kasein + Milchzucker + Salzen) erhaltenen, auf ein bestimmtes Volumen aufgefülten Fettlösung.

§) Oder man verfährt folgendermaßen:

$5 \mathrm{~g}$ Butter werden in ejner Porzellanschale geschmolzen und mit $20 \mathrm{~g}$ Gips gemischt, dann 6 Stunden lang bei etwa $100^{\circ}$ getrocknet und das nach dem Erkalten erhaltene trockene Pulver mit absolutem Äther im Extraktionsapparate bis zur Erschöpfung extrahiert.

Duclaux ${ }^{2}$ ), Hefelmann ${ }^{3}$ ), Fleischmann ${ }^{4}$ ) und G. Schüle $e^{5}$ ) bringen Verfahren der Fettbestimmung in Butter in Vorschlag, auf die hier nur verwiesen sei und die ebenfalls umständlich und zeitraubend sind. Dasselbe kann man von dem von dem Italiener Umberto Morin $i^{6}$ ) abgeänderten und vereinfachten Duclaux'schen Verfahren behaupten.

H. Lührig und F. Wiedemann ${ }^{7}$ ) haben ein Verfahren zur gleichzeitigen Bestimmung von Wasser, Salz und Fett in der Butter beschrieben, nach welchem sie etwa 2-3 g Butter in unten mit einigen Lagen Filtrierpapier geschlossenen und mit entfetteten Papierschnitzeln gefüllten Glashülsen von diesen aufsaugen lassen und nach vierstündigem Trocknen mit wasserfreiem Äther erschöpfen. Das in den "Vereinbarungen" angegebene direkte Verfahren der Fettbestimmung, sowie das von U. Morini verbesserte Duclaux'sche und das von Fleischmann vorgeschlagene Verfahren haben neben ihrer Umständlichkeit auch noch den Nachteil, daß die in Arbeit genommene Buttermenge eine viel zu grobe ist, sodaß bei dem nachherigen Trocknen des Fettes im Kolben nie der Zeitpunkt mit Sicherheit zu treffen ist, wann eine wirkliche Grewichtskonstanz eintritt, und unter Umständen ein recht langes Trocknen dazu erforderlich ist. Hierdurch tritt oft ein Bräunen des Fettes, bezw. eine Oxydation desselben, mithin eine Gewichtszunahme ein.

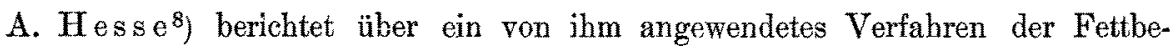

1) „Vereinbarungen “ Heft I, S. 95.

$\left.{ }^{2}\right)$ Stohmann, Milch und Molkereiprodukte 1898, S. 687 und Duclaux, Principes de laiterie, Paris, S. 111 und 258.

3) Zeitsehr. analyt. Chem. 1896, 35, 101.

4) Fleisehmann, Lehrbueh der Milchwirtschaft.

5) Chem.Ztg. 1902, 26, 653 .

$\left.{ }^{6}\right)$ Staz. sperim. agrar. Ital. 1899, 32, 517; diese Zeitschrift 1900, 8, 643.

7) Diese Zeitschrift 1904, 8, 247.

$\left.{ }^{8}\right)$ Diese Zeitschrift 1904, 8, 673-675. 
stimmung in Butter unter Anwendung des Gottlieb'schen Verfahrens zur Fettbestimmung in Milch.

$\mathrm{Zu}$ diesen Ausführungen ist zu bemerken, daß in der Versuchsstation für Molkereiwesen in Kiel schon seit 1900 die Fettbestimmungen in Butter nach Gottlieb ausgeführt werden, wenn auch in etwas anderer Art wie sie Hesse ausführt. Die Abweichungen sind folgende:

1. Hesse verwendet zum Abwägen der Butter kleine gespaltene, dünnwandige Glasröhrchen von etwa $3 \mathrm{~cm}$ Länge, streicht diese mit Butter voll, läßt sie in den Röse'schen Cylinder gleiten und gibt soviel heißes Wasser hinzu, bis die Gesamtmenge etwa $10 \mathrm{ccm}$ ausmacht. Nach dem Abkühlen wird dann wie gewöhnlich verfahren. Auch im hiesigen Institute wurden früher kleine, beiderseits offene Glasröbrchen verwendet, von deren Gebrauch ist aber wieder abgesehen worden, da beim Schütteln sehr oft der Boden der Cylinder durchgestoßen wurde; auferdem war das Füllen der Röhrchen ziemlich schwierig.

2. Nach dem hiesigen Verfahren wird nur zweimal, und zwar nach Vorschrift nacheinander, mit Äther und Petroläther aufgefüllt und in zwei verschiedene Kolben abgehebert und gewogen. Zweimaliges Auffüllen ist vollständig genügend, was bei der Besprechung des Verfahrens bewiesen werden wird.

3. Die angewendete Buttermenge ist womöglich noch kleiner als bei Hesse.

4. In der Herstellung der Durchschnittsprobe aus der zur Untersuchung gelangenden Butter wird anders verfahren.

Im Nachstehenden möge eine Besprechung der Anwendung des Gottlieb'schen Verfahrens zur Bestimmung des Butterfettes folgen:

Zwecks Erzielung einer guten Durchschnittsprobe für die Bestimmung von Wasser, Salz oder Fett wird die zur Untersuchung gelangende Butter in ein entsprechend großes Gefäß mit hermetisch schließendem Glasstöpsel gegeben, bei gelinder Wärme geschmolzen und darauf bis zum Erstarren unter öfterem Abkühlen kräftig geschüttelt. Butterfett und Serum werden auf diese Weise aufs innigste gemischt und ist ein Verdunsten von Wasser völlig ausgeschlossen. Die „Vereinbarungen" lassen für die Bestimmung des Wassers, dessen Rückstand sie ja für die Bestimmung des Fettes verwenden, eine Durchschnittsprobe derart nehmen, daß von möglichst vielen Stellen des Stückes mit einem blanken Messer, ohne Anwendung starken Druckes, dünne Scheiben vom ganzen Stück abgetrennt und über den Schalenrand abgestrichen werden. Es ist sehr zweifelhaft, ob auf diese Weise eine vollkommen genügende Durchschnittsprobe gewonnen wird. In vielen Laboratorien wieder wird die Butter in einem Mörser verrieben, wozu sie schon angewärmt werden muß, da die Wassertropfen in der kalten, festen Butter sonst nicht genügend verteilt werden. Man erzielt zwar beim kalten Mischen eine genügende Durchmischung, begünstigt aber gleichzeitig den Wasseraustritt aus der Butter, der um so beträchtlicher wird, je größer die Oberfläche der Butter im Mörser ist und je länger das Verreiben dauert. Außerdem kann noch Wasserverlust dadurch eintreten, daß bei nur geringer unvorsichtiger Handhabung des Pistills oder zu starkem Drücken Wassertropfen aus dem Mörser spritzen. Eine weitere Fehlerquelle bietet das Abwägen der in Arbeit genommenen Menge an der Luft.

Von der wie vorhin erwähnt vorbereiteten Butter werden etwa $3 \mathrm{~g}$ in ein bedecktes Wägegläschen gegeben, dasselbe gewogen und eine Spatelspitze voll, etwa 1,0-1,3 g, davon entnommen, und das Gläschen wieder gewogen. Auf diese Weise ist bequem und leicht das Gewicht der angewendeten Buttermenge festzustellen, ohne 
daß eine Verdunstung von Wasser möglich ist. Bei einiger Übung kann man ziemlich genau die vorhin erwähnte Buttermenge von etwa 1,0-1,3 g mit einem Spatel aus dem Wägegläschen entnehmen. Die durch Differenzwägung festgestellte Buttermenge spült man nun mit heißem Wasser - es genügen etwa $10 \mathrm{ccm}$ - durch einen kleinen Trichter mit kurzem Hals in einen Röse'schen Cylinder, kühlt ab, füllt mit $1 \mathrm{ccm}$ Ammoniak auf, schüttelt vorsichtig mit aufgesetztem Trichter durch, spült Trichter und Spatel mit $10 \mathrm{ccm}$ Alkohol ab, schüttelt wieder wie vorher und spült schlieBlich Spatel und Trichter noch mit $25 \mathrm{ccm}$ Äther ab. Weiter verfährt man wie gewöhnlich bei dem Gottlieb'schen Verfahren. Wendet man zuviel Wasser an, so scheidet sich die Ätherfettlösung schwer von der Serumschicht ab und beide Lösungen bleiben zum Teil emulgiert. Ferner muß zwischen Trichterhals und der Wandung des Cylinders ein Drahtende oder dergleichen eingefügt werden, um der Luft freien Abzug aus dem Cylinder zu verschaffen und etwaiges Spritzen der Flüssigkeiten zu vermeiden.

Nach etwa 4-stündigem Stehen ist die Fettlösung vollkommen klar und wird in einen kleinen gewogenen Erlenmeyer-Kolben abgehebert, das Heberrohr mit $25 \mathrm{cem}$ Ather in den Cylinder hinein abgespült und darauf Petroläther durch dasselbe in den Erlenmeyer-Kolben geblasen, wodurch es vollkommen von anhaftendem Fett befreit wird. Der Cylinder, in dem sich schon $25 \mathrm{ccm}$ Ather - von Abspülen des Heberrohrers her — befinden, wird wieder geschüttelt, dann $25 \mathrm{ccm}$ Petroläther zugegeben, ungeschïttelt und nach wieder etwa 4-stündigem Stehen die Ätherfettlösung wie vorher in einen zweiten gewogenen Erlen meyer-Kolben gehebert. Bei der zweiten Abheberung muß man aber die Flüssigkeitsmenge ablesen und daraus und aus der Gewichtszunahme des zweiten Kolbens die wirklich noch im Cylinder befindliche Fettmenge berechnen. Hesse dagegen gibt gleich nach dem ersten Abhebern $50 \mathrm{ccm}$ Äther zu und zieht sofort wieder in denselben Kolben ab, sodann schüttelt er mit einem Gemisch von $25 \mathrm{ccm}$ Äther $+25 \mathrm{ccm}$ Petroläther und hebert die Ätherfettschicht nach dem Klarwerden wieder in denselben Kolben ab. Das dritte Abhebern ist vollständig überflüssig, wenn man, wie vorher angegeben, verfährt. Bei einem Versuch, bei dem zum dritten Male vorschriftsmäßaig aufgefüllt und abgehebert war, wurde bei der Wägung erhalten an Fett $=0,0017$ und $0,0015 \mathrm{~g}$, während nach der Berechnung aus dem Volumen der zweiten Abheberung 0,0011 und $0,0017 \mathrm{~g}$ darin sein sollten, also sehr geringe Differenzen, die auf das Gesamtergebnis nur einen verschwindend geringen Einfluß haben. Die wach dicsem Verfahren ausgeführten Fettbestimmungen lieferten folgende Ergebnisse:

\begin{tabular}{r|c|c|c|c|c}
\hline $\begin{array}{c}\text { Ver- } \\
\text { such } \\
\text { No. }\end{array}$ & $\begin{array}{c}\text { Ergebnisse der } \\
\text { Doppelbestimmungen }\end{array}$ & $\begin{array}{c}\text { Differenz } \\
\text { zwischen beiden } \\
\text { Bestimmungen } \\
\% / 0\end{array}$ & $\begin{array}{c}\text { Ver- } \\
\text { such } \\
\text { No. }\end{array}$ & $\begin{array}{c}\text { Ergebnisse der } \\
\text { Doppelbestimmungen }\end{array}$ & $\begin{array}{c}\text { Differenz } \\
\text { zwischen beiden } \\
\text { Bestimmungen } \\
0 \%\end{array}$ \\
\hline I & 84,$50 ; 84,54$ & 0,04 & VI & 82,$30 ; 82,28$ & 0,02 \\
II & 84,$74 ; 84,72$ & 0,02 & VII & 84,$05 ; 84,05$ & 0 \\
III & 83,$85 ; 83,78$ & 0,07 & VIII & 84,$52 ; 84,50$ & 0,02 \\
IV & 85,$16 ; 85,05$ & 0,11 & IX & 81,$86 ; 81,85$ & 0,01 \\
V & 84,$22 ; 84,14$ & 0,12 & X & 84,$92 ; 84,86$ & 0,06
\end{tabular}

Wie aus der vorstehenden Tabelle ersichtlich ist, stimmen die Doppelbestimmungen unter sich sehr gut überein. Die größte Differenz beträgt $0,12 \%$, die mitt- 
lere Differenz aus den zehn Bestimmungen $0,047 \%$, Ergebnisse, mit denen auch ein anspruchsvoller Analytiker zufrieden sein kann. Die Gottlieb'sche Methode der Fettbestimmung in Milch läßt sich, mehr oder weniger modifiziert, mit Vorteił auf sämtliche Erzeugnisse des Molkereibetriebes ausdehnen, wie sie auch schon an vielen milchwirtschaftlichen Versuchsstationen seit Jahren als genauestes und sicherstes Verfahren der Fettbestimmung in Milch u. s. w. angewendet wird. Weibull ${ }^{1}$ ) machte schon im Jahre 1898 den Vorschlag, das Gottlieb'sche Verfahren als Einheitsmethode für Milchfettbestimmungen einzuführen, ein Vorschlag, dem sich $\mathrm{H}$. Weigmann wenigstens mit bezug auf die Fettbestimmung von Centrifugenmagermilch anschlob, und die Versuchsstation für Molkereiwesen in Kiel hat sich, wie die nitgeteilten Arbeiten erweisen, bemüht, die Anwendung des Gottlieb'schen Verfahrens anch auf die Erzeugnisse der Milch soviel wie möglich auszudehnen.

Hesse empfiehlt sie sogar als Universalmethode für sämtliche Molkereierzeugnisse, eine Ansicht, der man ruhig beistimmen kann.

1) Diese Zeitschrift 1898, 1, 413.

\title{
Quantitative Arsenbestimmung für forensisch-chemische Zwecke.
}

\author{
Von
}

\section{Mai.}

Mitteilung aus dem Laboratorium für angewandte Chemieder Universität München ${ }^{1}$ ).

Als Vorbedingung für den Nachweis und insbesondere für die quantitative Bestimmung des Arsens in Leichenteilen u. s. w. galt bisher die vollkommene Zerstörung der organischen Substanz.

An Verfahren hierzu ist bekanntlich zwar kein Mangel, doch zeichnet sich keines davon durch besondere praktisehe Brauchbarkeit und Annehmlichkeit in der Ausführung aus; sie sind im Gegenteil alle mehr oder weniger umständlich und zeitraubend, oder sonstwie nicht einwandfrei.

Am meisten benutzt wird wohl die Einwirkung von naszierendem Chlor aus Salzsäure und Kaliumchlorat nach v. Babo-Fresenius, ein Verfahren, dem indessen verschiedene Măngel anbaften; einmal sind zưr Zerstörung größerer Materialmengen, wie sie in der gerichtlichen Praxis meist vorliegen, sehr große Mengen Salzsäure und Kaliumchlorat erforderlich, sodaß die Flüssigkeiten sich öfters in einen Krystallbrei von Chlorkalium verwandeln und andererseits ist das Arbeiten damit überhaupt nicht gerade zu den angenehmen Operationen zu rechnen und die entweichenden Mengen freien Chlors erfordern eine außerordentlich gut wirkende Abzugsvorrichtung.

Ein weiter vielfach geübtes Verfahren beruht auf der Anwendung von Salpetersäure mit oder ohne Zusatz von Schwefelsäure; auch hierbei sind zur völïigen Zer-

1) Die Mitteilung war auf der am 2. und 3. Juni d. J. in Dresden stattgehabten Versammlung der Freien Vereinigung Dentscher Nahrungsmittelchemiker zum Vortrag bestimmt, der leider damals ausfallen mufite. 